\title{
Comparison of total, salivary and calculated free cortisol levels in patients with severe sepsis
}

\author{
Gulsah Elbuken ${ }^{1}$, Zuleyha Karaca ${ }^{1}$, Fatih Tanriverdi' ${ }^{1}$ Kursad Unluhizarci ${ }^{1}$, Murat Sungur ${ }^{2}$, Mehmet Doganay ${ }^{3}$ \\ and Fahrettin Kelestimur ${ }^{\text {* }}$
}

\begin{abstract}
Background: The purposes of the study were to compare serum total cortisol (STC), salivary cortisol ( $\mathrm{SaC}$ ) and calculated free cortisol (CFC) levels at baseline and after the adrenocorticotrophic hormone (ACTH) stimulation test in patients with severe sepsis (SS) and determine the suitability of use of $\mathrm{SaC}$ and CFC levels instead of STC for the diagnosis of adrenal insufficiency (AI) in patients with SS. And secondary aims of this study were to compare these parameters in patients with SS with healthy controls and check their effects on survival status of the patients.

Methods: Thirty patients with SS (15 men and 15 women) were compared with 16 healthy controls. Low-dose $(1 \mu \mathrm{g}) \mathrm{ACTH}$ stimulation test was performed to the patients on the first, seventh and 28th days of diagnosis of SS, but in control group, $1 \mu \mathrm{g} \mathrm{ACTH}$ stimulation test was performed only once. STC, SaC and CFC levels were measured during ACTH stimulation test.

Results: Patients were categorized as having low or high baseline STC according to a cut-off level of $10 \mu \mathrm{g} / \mathrm{dL}$. In high STC group, baseline and peak SaC levels were found to be $2.3(0.2-9.0)$ and $3.4(0.5-17.8) \mu \mathrm{g} / \mathrm{dL}$ on D1 and 1.1 $(0.8-4.6)$ and $2.6(1.3-2.9) \mu \mathrm{g} / \mathrm{dL}$ on D7, respectively. In the control group, baseline and peak SaC levels were 0.4 $(0.1-1.4)$ and $1.1(0.4-2.5) \mu \mathrm{g} / \mathrm{dL}$, respectively. Baseline and peak SaC levels after ACTH stimulation were found to be higher in high STC group than in controls, but they were found to be similar in low STC and control groups. In high STC group, CFC levels were $0.3(0.1-0.3)$ and $0.4(0.3-0.7) \mu \mathrm{g} / \mathrm{dL}$ on D1 and $0.2(0.1-0.3)$ and $0.4(0.1-0.7) \mu \mathrm{g} / \mathrm{dL}$ on D7, respectively. In the control group, baseline and peak cFC levels were $1.7(0.4-1.9)$ and $1.8(1.0-6.6) \mu \mathrm{g} / \mathrm{dL}$, respectively. CFC levels were found to be lower in patients with SS subgroups than in the control group. Baseline and stimulated STC, SaC and CFC levels did not differ according to the survival status. SaC, CFC and STC levels were found to be correlated with each other.

Conclusions: SS is associated with increased SaC, but decreased CFC levels when baseline STC is assumed to be sufficient. When STC level is assumed to be insufficient, SaC levels remain unchanged, but CFC levels are decreased. Lower STC levels is not associated with increased mortality in patients with SS. More data are needed in order to suggest the use of SaC and CFC instead of STC.
\end{abstract}

Trial registration: ClinicalTrials.gov No: NCT02589431

Keywords: ACTH stimulation test, HPA axis, Total cortisol, Salivary cortisol, Calculated free cortisol, Severe sepsis

\footnotetext{
* Correspondence: fktimur@erciyes.edu.tr

${ }^{1}$ Department of Endocrinology and Metabolism, Erciyes University Medical

School, 38039 Kayseri, Turkey

Full list of author information is available at the end of the article
} 


\section{Background}

In stress-free people, cortisol is secreted in diurnal pattern. Any type of acute illness or trauma results in loss of the diurnal variation of cortisol secretion [1]. In case of a critical illness, organism needs to maintain adequate cortisol levels to cope with the illness. Cortisol response to such an illness is one of the factors that determines survival [2]. Lower or higher levels of serum total cortisol (STC) levels than expected were reported to be closely related to survival status [3]. As a kind of critical illness, sepsis may lead to major changes in hypothalamo-pituitaryadrenal (HPA) axis that causes challenges in the evaluation of its functions in those patients $[4,5]$.

Relative adrenal insufficiency (RAI), or critical illnessrelated corticosteroid insufficiency (CIRCI), is a form of adrenal insufficiency (AI) in critically ill patients whose cortisol levels are inadequate for the severe stress response they experience [6]. The diagnosis of CIRCI and the indications for corticosteroid therapy in critical illness $(\mathrm{CI})$ are still controversial [3, 6-10]. Baseline or random STC levels, cortisol increment after adrenocorticotrophic hormone (ACTH) stimulation test (delta cortisol), free cortisol (FC) levels, or other markers presenting FC, or a combination of these tests have been proposed as diagnostic tests for CIRCI [4, 8-11].

Annane et al. have reported a delta cortisol of less than $9 \mu \mathrm{g} / \mathrm{dL}$ or a random cortisol of less than $10 \mu \mathrm{g} / \mathrm{dL}$ as the best predictors of AI in patients with severe sepsis (SS) and septic shock [9]. But, in critically ill patients, since the adrenal glands might already be maximally stimulated, baseline cortisol levels may be higher than ACTH-stimulated serum cortisol levels [4, 5]. For this reason, some authors suggest measuring random serum cortisol levels at any time of the day regardless of cortisol diurnal rhythm $[6,9]$.

More than $90 \%$ of circulating cortisol is predominantly bound to not only cortisol binding globulin (CBG) but also albumin [12]. Thus, in the presence of both hypoalbuminemia and decreased CBG levels, the ratio of bound to free cortisol levels can be altered. In this situation, measurement of FC becomes more important. Since direct FC measurement is time consuming and non-automated, some indirect methods to determine FC levels had been introduced such as Coolens' method [12, 13]. In patients with CI, the synthesis of CBG and albumin is reduced leading to overestimation of $\mathrm{AI}$ if we only use STC levels. Since some studies demonstrated that salivary cortisol $(\mathrm{SaC})$ reflects free or unbound plasma cortisol levels, this method is used more often in clinical studies [14-19]. STC and SaC levels at baseline and after ACTH stimulation had been used in some studies in patients with CI $[17,18]$.

The aims of the present study were to compare STC, $\mathrm{SaC}$ and calculated free cortisol (cFC) levels at baseline and after the ACTH stimulation test in patients with SS and determine the suitability of $\mathrm{SaC}$ and cFC levels instead of STC for the diagnosis of AI in patients with SS. And secondary aims of this study were to compare these parameters in patients with SS with healthy controls and check their effects on survival status of the patients.

\section{Patients and methods}

The study was registered to Clinical Trials. Clinical trial Gov No: NCT02589431.

\section{Patients}

Patients meeting the American College of Chest Physicians (ACCP)/Society of Critical Care Medicine (SCCM) consensus definition of SS [20] were enrolled from the Internal Medicine Intensive Care Unit (ICU) of our hospital. The study was approved by the Local Ethics Committee. Informed consent was obtained from all volunteers or their first-degree relatives. Exclusion criteria were the presence of malignancy, history of corticosteroid exposure, and also presence of any disease that could affect HPA axis. We also excluded the patients who have any visible bleeding in their oral cavity.

Thirthy patients with SS (15 men and 15 women) and 16 (8 men and 8 women) healthy controls were included in the study. The first $24 \mathrm{~h}$ of diagnosis of SS was shown as day (D) 1. Baseline hormone tests were obtained especially in the morning between 08.00-09.00 a.m. within $24 \mathrm{~h}$ after the symptoms of SS were detected (D1). HPA axis was evaluated by baseline STC, SaC and cFC levels, and STC, SaC and $\mathrm{cFC}$ response to low-dose $(1 \mu \mathrm{g})$ ACTH stimulation test. ACTH stimulation test was also performed in the morning. The patients who were alive and accepted to continue in the participation of the study were evaluated again on seventh (D7) and 28th (D28) days of SS.

While the patients were treated for SS by his/her own physician in ICU, they were evaluated for the presence/ absence of (relative) AI concurrently. AI was suspected/ considered clinically in the presence of hemodynamic instability and/or hyponatremia-hyperkalemia. Patients, who were determined as AI by their treating physician and administered glucocorticoid therapy, were excluded from the study.

Apart from routine daily evaluations, blood samples were obtained from all the patients in accordance with the study protocol. All the blood and saliva samples were stored at $-80{ }^{\circ} \mathrm{C}$ and assayed after the study protocol finished. Thus, although this is a prospective study, samples were analysed retrospectively.

Low-dose ACTH stimulation test was performed on D1, D7 and D28 as described previously [21]. cFC levels were estimated by Coolens' equation [13]. Blood and saliva samples were obtained before and 30 and $60 \mathrm{~min}$ 
after ACTH injection. STC, CBG and SaC levels were measured during the $\mathrm{ACTH}$ stimulation tests. We have used baseline and peak hormone levels after ACTH stimulation test to compare the groups. No adverse events were reported during the tests. Between D1-D7 of the study, only baseline STC, $\mathrm{SaC}$ and cFC levels were obtained. Acute Physiology and Chronic Health Evaluation (APACHE) II score [22] and Sequential Organ Failure Assessment (SOFA) score [23] were calculated for each patient on D1, D7 and D28. Data were compared with 16 healthy controls. Patient enrollment is summarized in Fig. 1.

Irrespective of the decision of the treating physician on the diagnosis of AI, we have categorized the patients retrospectively as low or high baseline STC group according to the baseline STC cut-off level of $10 \mu \mathrm{g} / \mathrm{dL}$ on D1, D7 and D28.

\section{Assays}

STC levels were measured by RIA method (Immunotech; Prag, Czech Republic) with an intraassay coefficient of variation $5.1 \%$ and interassay coefficient of variation $9.2 \%$. Serum CBG concentrations were analysed with RIA (BioSource Europe S.A., Nivelles, Belgium). Intraassay and interassay coefficients of variation were 3.9 and $5.5 \%$, respectively. The Coolens' method was used to calculate serum FC concentration: $\mathrm{FC}^{2} K(1+N)+\mathrm{FC}[1$ $+N+K(G-T)]-T=0$, where $K=3 \times 10^{-7} \mathrm{M}^{-1}, \mathrm{M}=$ affinity of $\mathrm{CBG}$ to cortisol at $37{ }^{\circ} \mathrm{C}, G=\mathrm{CBG}, \mathrm{FC}=$ free cortisol, $T=$ cortisol and $N=$ ratio of albumin bound to FC (1.74). FC was calculated as follows: $F C=$ $\sqrt{Z^{2}+\frac{T}{(1+N) K}-Z}$, and $Z=\frac{1}{2 K}+\frac{G-T}{2(1+N)}$ [13]. Saliva samples were collected, stored and analysed as described previously [24]. SaC was measured by using highsensitivity enzyme immunassay (EI) kit (Salimetrics ${ }^{\circ}$ Inc, State College, PA, USA), according to the manufacturer's instructions $[15,17]$. The interassay coefficient of variation over the range of low to high values varied from 5.7 to $6.8 \%$, whereas the respective intraassay coefficients of variation were 3.2 and $6.3 \%$ [15].

\section{Statistical analysis}

All statistical analysis were done by Statistical Package for Social Sciences (SPSS for Windows, version 15; Chicago; IL). Normal distribution of the data were tested by Shapiro-Wilk test. Since the data were not distributed normally, statistical analysis was done by nonparametric tests. The hormone results are presented as median, minimum and maximum levels. Statistical significance was set at $p$ value less than 0.05 .

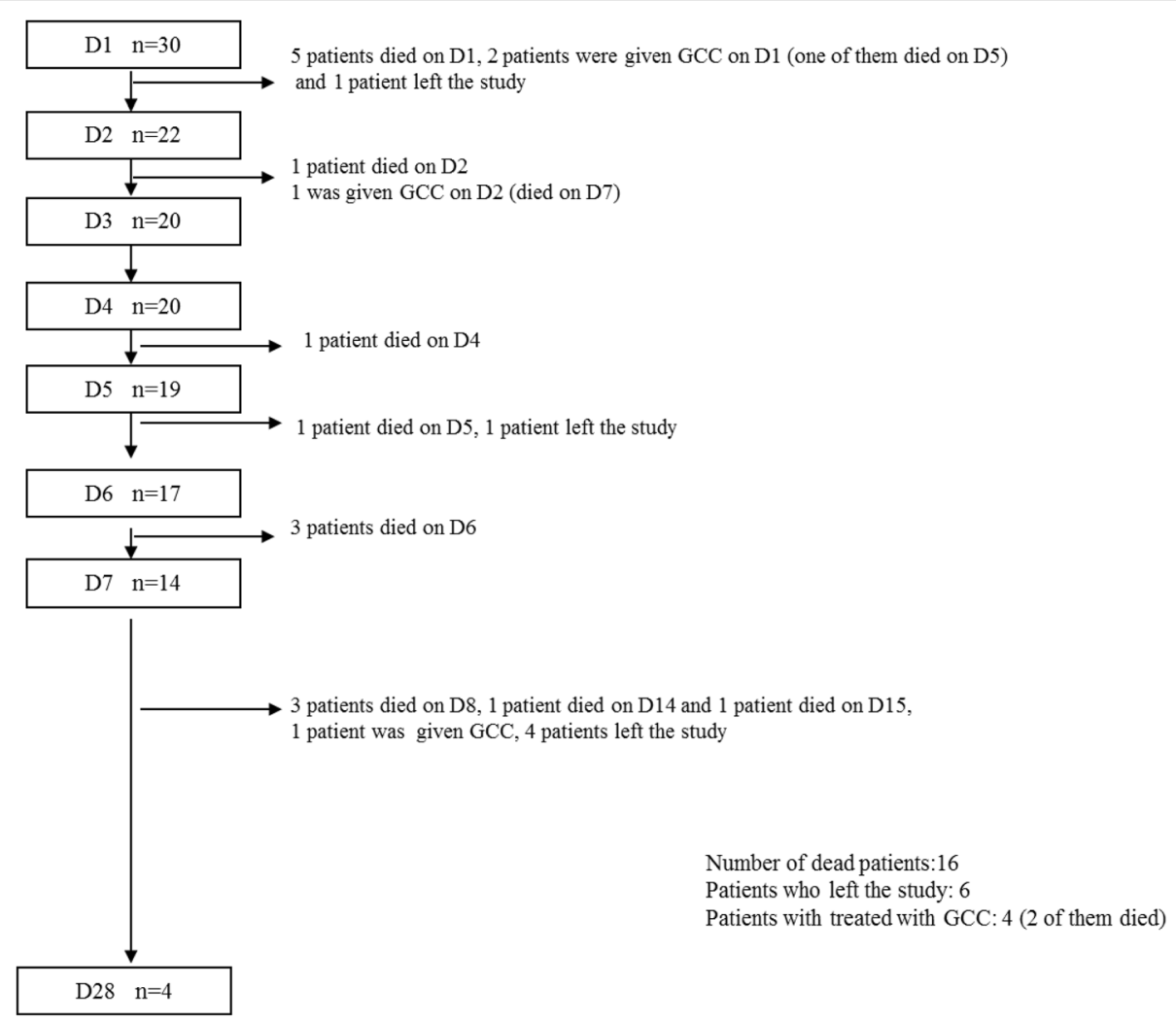

Fig. 1 Patient enrollment 


\section{Results}

There were no statistical difference between the mean ages of the patients of SS and the control group (61.7 \pm 14.7 (range 23-82) and 58.7 \pm 4.1 (range 51-64) years, respectively).

Among patients diagnosed with SS, the infection originated from the lung in 12 patients (40\%) (pneumonia), blood in 4 patients (13\%), urine in 8 patients (27\%), gastrointestinal tract in 4 patients $(13 \%)$ and skin and soft tissue in 2 patients $(7 \%)$. Mean leukocyte count of SS patients was $12,940 \pm 7502 / \mu \mathrm{L}$.

Comorbid conditions in these 30 SS patients included type 2 diabetes mellitus (DM) in 6 patients (20\%), coronary heart disease in 11 patients (37\%) and chronic obstructive pulmonary disease (COPD) in 4 patients (10\%). Nine patients (30\%) did not have any comorbidity. No remarkable comorbidity was present in control subjects.

Among 21 patients with comorbidities, 5 were using metformin, 2 were using metformin and gliclazide, 6 were using angiotensin converting enzyme inhibitors or angiotensin II receptor blockers and 1 was using an angiotensin II receptor blocker and a beta blocker combination therapy before development of SS. All of the patients with COPD were using short-acting beta- 2 agonists, and none of them was using inhaled corticosteroids. Due to hypotension, the antihypertensive drugs were not administered to patients during SS. The patients with DM were followed with subcutaneous insulin treatment, and treatment regimens other than glucocorticoids inhaled treatments were administered to patients with COPD.

At the onset of SS, all 30 patients were found to have reduced blood pressure (100\%) and additionally hypothermia occurred in 5 patients (17\%), hyperkalemia in 2 patients $(7 \%)$ and hyponatremia in 4 patients (13\%). Growth of microorganisms was observed in body fluid cultures in 22 patients (\%73) and 10 patients (33\%) required vasopressor infusions. A total of 4 patients (13\%) were administered glucocorticoid therapy according to the judgement of following physician. These 4 patients were excluded from further analysis in the study after steroid administration.

Patients were categorized as low or high STC group according to their baseline STC (low if STC $<10 \mu \mathrm{g} / \mathrm{dL}$, high if STC $\geq 10 \mu \mathrm{g} / \mathrm{dL}$ [9].

Thirty patients were included in the study on D1 and 11 patients were defined as low STC according to their baseline STC levels on D1. Low STC group had delta STC levels $<9 \mu \mathrm{g} / \mathrm{dL}$ and peak STC levels $<21 \mu \mathrm{g} / \mathrm{dL}$ on D1. Not only baseline STC but also peak STC, peak SaC and basal and peak cFC levels were also found to be significantly lower in patients with low STC group. Baseline and stimulated CBG levels on D1 were not different in two groups (Table 1).
All of 5 patients died on D1 had high STC levels (41, $21,25,32$, and $31 \mu \mathrm{g} / \mathrm{dL}$, respectively). Five of $11 \mathrm{pa}-$ tients in low STC group died and 6 survived until D7. Among 6 patients who survived until D7, 1 patient had baseline STC levels 8.4 and $12.4 \mu \mathrm{g} / \mathrm{dL}$ on D1 and D7, respectively. The remaining 5 patients with low STC on D1 had also low STC on D7. Newly onset low STC levels were present in 5 patients on D7. Baseline cFC levels were also found to be lower in low STC group (Table 1). Baseline or peak $\mathrm{SaC}$ and cFC levels were not found to be different in low or high STC groups (Table 1).

No marked difference was observed between low or high STC groups in means of albumin values and APACHE II and SOFA scores. However, albumin levels were significantly lower compared to healthy group irrespective of the baseline STC $(p<0.01)$. Groups were compared at the end of D7 and D28 with respect to serum albumin levels obtained on D1 and D7 depending on survival. Serum albumin values did not differ between SS subgroups. Mean APACHE II score was 21 (min 7, $\max 37)$ on $D 1$ and $12(\min 7, \max 29)$ on D7 among survivors and $32(\min 16, \max 40)$ on D1 and 33 (min 25 , max 36) on D7 among non-survivors $(p<0.01)$. Mean SOFA score was $6(\min 1, \max 12)$ on D1 and 4 (min 1, max 6) on D7 among survivors and 11 (min 3, $\max 21)$ on $\mathrm{D} 1$ and $13(\min 8, \max 14)$ on D7 among non-survivors $(p<0.01)$.

Hormone levels between D1-D7 were compared in patients with SS who have survived or not until D7. On D5 and D7, baseline STC and cFC levels and, on D1, baseline STC and baseline and peak cFC levels were found to be lower in patients who have survived. There were no differences between $\mathrm{SaC}$ and CBG levels of the patients who have survived or not (Table 2). The patients with low baseline STC on D1 $(n=11)$ were divided into two groups according to their survival status until D7. Baseline and stimulated STC, SaC and cFC levels were not significantly different in patients who have survived or not. Similarly, if 19 patients with high baseline STC on D1 were divided into two groups according to their survival status until D7, baseline and stimulated STC, SaC and CFC levels were not found to be different in patients who have survived or not (data not shown).

In total, 11 patients died before D7, 3 patients were treated with glucocorticoid between D1 and D7 (and 2 of them died before D7); 5 patients died before D28, 1 was given glucocorticoid between D7 and D28. At the end of 28 days, 8 patients were alive.

Nineteen patients had high STC on D1, 3 of them were given glucocorticoids (2 died), 6 of them died, 2 of them left the study and 8 were alive at the end of 7 days. Four patients had high STC on D7, 2 died, 1 were given glucocorticoid and 1 were alive at the end of 28 days. 
Table 1 Baseline and peak CBG, CFC, SaC and STC levels on D1 and D7

\begin{tabular}{|c|c|c|c|c|c|c|c|c|c|c|c|c|c|c|c|c|}
\hline \multicolumn{2}{|c|}{ Day of the study } & \multicolumn{3}{|l|}{ D1 } & \multicolumn{3}{|l|}{ D7 } & \multirow[t]{2}{*}{ Healthy } & \multirow[t]{2}{*}{$p 1$} & \multirow[t]{2}{*}{$p 2$} & \multirow[t]{2}{*}{ p3 } & \multirow[t]{2}{*}{$p 4$} & \multirow[t]{2}{*}{ p5 } & \multirow[t]{2}{*}{ p6 } & \multirow[t]{2}{*}{ p7 } & \multirow[t]{2}{*}{$p 8$} \\
\hline & & All SS patients & SS with high STC & SS with low STC & All SS patients & SS with high STC & SS with low STC & & & & & & & & & \\
\hline$n$ & & 30 & 19 & 11 & 14 & 4 & 10 & 16 & & & & & & & & \\
\hline \multirow[t]{2}{*}{ STC ( $\mu \mathrm{g} / \mathrm{dL})$} & Baseline & $13.7(3.1-53.2)$ & $18.7(10.4-53.2)$ & $8.2(3.1-9.4)$ & $7.6(0.4-13.9)$ & $12.4(12.0-13.9)$ & $6.5(0.4-9.6)$ & $14.3(4.3-20)$ & 0.13 & $<0.01$ & $<0.01$ & $<0.01$ & $<0.01$ & 0.01 & 0.67 & 0.03 \\
\hline & Peak & $20.3(7.4-52.0)$ & $31.0(14.1-52.0)$ & $11.0(7.4-20.6)$ & $12.7(4.0-25.0)$ & $18.9(8.3-25.0)$ & $12.4(3.9-21.4)$ & $27(15.2-41.4)$ & 0.64 & $<0.01$ & 0.27 & $<0.01$ & $<0.01$ & 0.24 & 0.02 & $<0.01$ \\
\hline \multirow[t]{2}{*}{$\mathrm{SaC}(\mu \mathrm{g} / \mathrm{dL})$} & Baseline & $1.9(0.2-9.0)$ & $2.3(0.2-9.0)$ & $0.9(0.2-2.9)$ & $0.7(0.3-4.6)$ & $1.1(0.8-4.6)$ & $0.6(0.3-3.0)$ & $0.4(0.1-1.4)$ & $<0.01$ & 0.15 & $<0.01$ & 0.09 & 0.04 & 0.07 & 0.06 & 0.72 \\
\hline & Peak & $2.2(0.5-17.8)$ & $3.4(0.5-17.8)$ & $1.9(0.6-4.2)$ & $1.7(0.2-4.0)$ & $2.6(1.3-2.9)$ & $1.5(0.2-4.0)$ & $1.1(0.4-2.5)$ & $<0.01$ & 0.02 & $<0.01$ & 0.11 & 0.37 & 0.39 & 0.01 & 0.14 \\
\hline \multirow[t]{2}{*}{$\mathrm{cFC}(\mu \mathrm{g} / \mathrm{dL})$} & Baseline & $0.5(0.1-1.9)$ & $0.7(0.4-1.9)$ & $0.3(0.1-0.3)$ & $0.3(0.1-0.5)$ & $0.4(0.4-0.5)$ & $0.2(0.1-0.3)$ & $0.7(0.4-1.9)$ & 0.48 & $<0.01$ & $<0.01$ & $<0.01$ & $<0.01$ & 0.01 & 0.65 & $<0.01$ \\
\hline & Peak & $0.71(0.3-1.9)$ & $1.1(0.5-1.9)$ & $0.4(0.3-0.7)$ & $0.4(0.1-0.9)$ & $0.7(0.3-0.9)$ & $0.4(0.1-0.7)$ & $1.8(1.0-6.6)$ & $<0.01$ & $<0.01$ & $<0.01$ & $<0.01$ & $<0.01$ & 0.24 & 0.89 & 0.12 \\
\hline \multirow[t]{2}{*}{ CBG $(\mu \mathrm{g} / \mathrm{dL})$} & Baseline & $0.3(0.1-0.6)$ & $0.3(0.1-0.6)$ & $0.2(0.1-0.4)$ & $0.3(0.2-0.5)$ & $0.4(0.3-0.4)$ & $0.3(0.2-0.5)$ & $0.5(0.3-0.8)$ & $<0.01$ & 0.12 & $<0.01$ & $<0.01$ & $<0.01$ & 0.94 & $<0.01$ & $<0.01$ \\
\hline & Peak & $0.3(0.1-0.5)$ & $0.3(0.1-0.5)$ & $0.3(0.1-0.5)$ & $0.4(0.1-0.5)$ & $0.3(0.2-0.4)$ & $0.4(0.1-0.5)$ & $0.6(0.4-0.9)$ & $<0.01$ & 0.6 & $<0.01$ & $<0.01$ & $<0.01$ & 0.35 & $<0.01$ & $<0.01$ \\
\hline
\end{tabular}

Abbreviations: D1 first day of severe sepsis, $n$ number of the subjects, STC serum total cortisol, SaC salivary cortisol, $c F C$ calculated free cortisol, CBG cortisol binding globulin, $p 1$ belongs to comparison between

hormon levels of the patients with severe sepsis on D1 and healthy control, $p 2$ belongs to comparison between patients who have basal STC $\geq$ or $<10 \mu \mathrm{g} / \mathrm{dL}$ on D1, $p 3$ belongs to comparison between patients with

severe sepsis who have baseline STC $\geq 10 \mu \mathrm{g} / \mathrm{dL}$ on D1 and healthy control, $p 4$ belongs to comparison between patients with severe sepsis who have baseline STC $<10 \mu \mathrm{g} / \mathrm{dL}$ on D1 and healthy control, $p 5$ belongs to

comparison between hormone levels of the patients with severe sepsis on D7 and healthy control, $p 6$ belongs to comparison between patients who have baseline STC $\geq$ or $<10 \mu \mathrm{g} / \mathrm{dL}$ on $\mathrm{D} 7, p 7$ belongs to

healthy control 
Table 2 Differences between STC, SaC, CFC and CBG levels of the patients who have survived or not

\begin{tabular}{|c|c|c|c|c|c|c|c|c|c|c|}
\hline \multirow[t]{2}{*}{ Days } & \multirow[t]{2}{*}{ Number } & \multirow[t]{2}{*}{ Survival } & \multicolumn{2}{|l|}{ STC $(\mu \mathrm{g} / \mathrm{dL})$} & \multicolumn{2}{|l|}{$\mathrm{SaC}(\mu \mathrm{g} / \mathrm{dL})$} & \multicolumn{2}{|l|}{$c F C(\mu \mathrm{g} / \mathrm{dL})$} & \multicolumn{2}{|l|}{ CBG $(\mu \mathrm{g} / \mathrm{dL})$} \\
\hline & & & Baseline & Peak & Baseline & Peak & Baseline & Peak & Baseline & Peak \\
\hline \multirow[t]{2}{*}{$\overline{\mathrm{D} 1}$} & 30 & Alive & $9.4(3.1-25.8)$ & $14.3(7.4-46.9)$ & $1.0(0.2-7.5)$ & $2.0(0.6-15.0)$ & $0.3(0.1-0.9)$ & $0.5(0.3-1.7)$ & $0.3(0.1-0.6)$ & $0.4(0.2-0.5)$ \\
\hline & & Dead & $21.0(4.6-53.2)$ & $31.0(7.5-52.0)$ & $2.3(0.2-9.0)$ & $3.4(0.5-17.8)$ & $0.8(0.2-1.87)$ & $1.1(0.3-1.9)$ & $0.2(0.1-0.5)$ & $0.3(0.1-0.5)$ \\
\hline \multirow[t]{2}{*}{ D2 } & 22 & Alive & $12.4(4.9-40.9)$ & & $0.7(0.1-3.3)$ & & $0.4(0.2-1.4)$ & & $0.2(0.1-0.5)$ & \\
\hline & & Dead & $17.7(5.6-24.8)$ & & $0.6(0.1-5.2)$ & & $0.8(0.3-1.5)$ & & $0.2(0.2-0.5)$ & \\
\hline \multirow[t]{2}{*}{ D3 } & 20 & Alive & $10.2(3.2-31.3)$ & & $0.7(0.1-3.8)$ & & $0.3(0.1-1.1)$ & & $0.2(0.1-0.5)$ & \\
\hline & & Dead & 22.8 (7.9-43.3) & & $0.9(0.1-5.2)$ & & $0.8(0.3-1.5)$ & & $0.2(0.2-0.4)$ & \\
\hline \multirow[t]{2}{*}{ D4 } & 20 & Alive & $12.6(3.5-22.1)$ & & $0.8(0.1-3.0)$ & & $0.5(0.1-0.8)$ & & $0.3(0.1-0.4)$ & \\
\hline & & Dead & $18.6(3.3-50.5)$ & & $2.2(0.2-9.4)$ & & $0.7(0.1-1.8)$ & & $0.3(0.1-0.4)$ & \\
\hline \multirow[t]{2}{*}{ D5 } & 19 & Alive & $11.4(5.2-30.4)$ & & $0.8(0.1-4.6)$ & & $0.4(0.2-1.1)$ & & $0.3(0.1-0.4)$ & \\
\hline & & Dead & $20.2(15.6-30.0)$ & & $0.7(0.1-3.1)$ & & $0.7(0.6-1.1)$ & & $0.2(0.2-0.4)$ & \\
\hline \multirow[t]{2}{*}{ D6 } & 17 & Alive & $10.4(2.1-43.0)$ & & $1.0(0.1-4.6)$ & & $0.4(0.1-1.5)$ & & $0.2(0.1-0.5)$ & \\
\hline & & Dead & $27.3(5.6-32.6)$ & & $2.0(0.9-4)$ & & $1.0(0.2-1.2)$ & & $0.3(0.1-0.4)$ & \\
\hline \multirow[t]{2}{*}{ D7 } & 14 & Alive & $6.9(0.4-13.9)$ & $9.9(3.9-25.0)$ & $0.5(0.3-3.0)$ & $1.7(0.2-4.0)$ & $0.2(0.1-0.5)$ & $0.4(0.1-0.9)$ & $0.3(0.2-0.5)$ & $0.4(0.2-0.5)$ \\
\hline & & Dead & $8.7(5.6-12.4)$ & $13.3(8.3-18.8)$ & $0.9(0.6-4.6)$ & $2.4(0.6-2.9)$ & $0.3(0.2-0.4)$ & $0.5(0.3-0.7)$ & $0.3(0.2-0.5)$ & $0.3(0.1-0.4)$ \\
\hline$p 1$ & & & 0.02 & 0.53 & 0.28 & 0.12 & 0.02 & 0.01 & 0.39 & 0.27 \\
\hline$p 2$ & & & 0.08 & & 0.64 & & 0.57 & & 0.56 & \\
\hline p3 & & & 0.55 & & 0.69 & & 0.06 & & 0.36 & \\
\hline$p 4$ & & & 0.02 & & 0.32 & & 0.55 & & 0.91 & \\
\hline p5 & & & 0.02 & & 0.9 & & 0.02 & & 0.44 & \\
\hline p6 & & & 0.3 & & 0.2 & & 0.3 & & 0.5 & \\
\hline$p 7$ & & & 0.01 & 0.24 & 0.07 & 0.39 & 0.01 & 0.24 & 0.94 & 0.35 \\
\hline
\end{tabular}

Abbreviations: STC serum total cortisol, SaC salivary cortisol, $c F C$ calculated free cortisol, CBG cortisol binding globulin, $D$ day of severe sepsis, $p 1, p 2, p 3, p 4, p 5, p 6$ and $p 7$ belongs to comparisons between patients with severe sepsis on D1, D2, D3, D4, D5, D6 and D7 who have survived or not 
Five of 11 patients with low STC on D1 died and 6 were alive at the end of 7 days. One of the patients with low STC group on D1 was recovered until D7. On D7, 5 new patients with baseline STC levels lower than $10 \mu \mathrm{g} /$ $\mathrm{dL}$ were added to lower STC group (final number was 10). Three of these 10 patients with low STC value on D7 died and 7 of them were alive at the end of 28 days. One of the 8 patients who were alive on D28 was in high STC group. Although 8 patients were alive, only 4 patients could participate our study on D28 of SS. The individual data of the these 4 patients on D28 are summarized in Table 3.

When patients were divided into two groups according to their survival status on D28, there were no differences in hormone levels of the patients between two groups. Only APACHE II and SOFA scores were found to be significanly higher in patients who died.

Data of patients with SS were compared with 16 healthy people. Baseline STC, baseline and stimulated $\mathrm{SaC}$ levels were significantly higher in patients with SS with high STC than in healthy subjects. Baseline and stimulated cFC levels were found to be higher in high STC group than in low STC SS groups. But baseline and stimulated cFC levels were found to be lower in patients with high STC on D1 than control group. On D7, only baseline cFC levels were found to be higher in patients with high STC than in patients with low STC. Baseline and stimulated STC and cFC levels were found to be significantly lower in patients with low baseline STC than in healthy people, but $\mathrm{SaC}$ levels were found to be similar in two groups (Table 1). Serum CBG and albumin levels were found to be significantly lower in both SS groups than in healthy group (Table 1).

All simultaneous baseline SaC and STC levels of patients with SS were pooled, and 145 pairs were obtained. Similarly, all simultaneous $\mathrm{SaC}$ and STC responses of patients to low-dose ACTH stimulation test were also pooled, and 48 pairs were obtained. SaC and STC were positively correlated with each other $(p<0.01, r=0.26$ and $p<0.01, r=0.30$, respectively, for random and stimulated levels). $\mathrm{SaC}$ and $\mathrm{cFC}$ levels were also positively correlated with each other $(p=0.02, r=0.23$ and $p<0.01, r=0.53$, respectively, for random and stimulated levels) (Fig. 2).

\section{Discussion}

In this study, baseline STC levels were found to be higher in high STC SS patients (suspected to have intact HPA axis) than in healthy subjects. This finding was supported by previous data $[25,26]$. Not only baseline STC but also peak STC, baseline and peak SaC responses after ACTH stimulation were found to be significantly higher in high STC group than in both low STC and control group. So, the cortisol production is increased in order to cope with the severity of sepsis.

It is known that an appropriate response of HPA axis to $\mathrm{CI}$ is crucial for survival; so, both very high or low responses have been associated with increased mortality $[27,28]$. In the present study, death was found to be associated with high cortisol levels. Many studies have demonstrated increased STC levels in sepsis, and the degree of the elevation is related to the severity of the illness [25-29]. Similarly, in a study by Yildiz et al., the mean baseline plasma cortisol concentration was significantly lower in survivors compared with non-survivors in sepsis [25]. In another, although baseline and stimulated STC levels were higher in sepsis group than their recovery status on the 14th day of sepsis, baseline and stimulated STC levels were not found to be related to mortality [29]. But in the present study, baseline and peak STC on D1 and baseline STC levels between D1D7 tended to be lower in patients who survived. Both baseline and stimulated STC levels were lower in the present study than in the study of Yildiz et al. [29]. In their study, higher peak STC levels may be explained by the use of higher dose of synthetic $\mathrm{ACTH}$ for test, but baseline STC levels were also found to be higher. Patients group which enrolled the study by Yildiz et al. included not only patients with SS but also sepsis [29]. So, their study population was not homogenous. Discordance between the two studies may be explained by this scenario.

In the present study, serum CBG concentrations were found to be lower in SS than in healthy individuals compatible with prior studies $[13,30]$. The lower CBG levels in sepsis can be explained by multifactorial causes such as decreased liver synthesis of CBG, proteolytic cleavage by neutrophil elastase, and less common genetic mutations

Table 3 Individual data of 4 patients on D28

\begin{tabular}{|c|c|c|c|c|c|c|c|c|c|c|c|c|c|c|}
\hline \multirow[t]{2}{*}{$\overline{\mathrm{No}}$} & \multirow[t]{2}{*}{ Age } & \multirow[t]{2}{*}{ Gender } & \multicolumn{2}{|c|}{ STC $(\mu \mathrm{g} / \mathrm{dL})$} & \multicolumn{2}{|c|}{$\mathrm{SaC}(\mu \mathrm{g} / \mathrm{dL})$} & \multicolumn{2}{|c|}{$\mathrm{cFC}(\mu \mathrm{g} / \mathrm{dL})$} & \multicolumn{2}{|c|}{ CBG $(\mu \mathrm{g} / \mathrm{dL})$} & \multirow{2}{*}{$\begin{array}{l}\text { Albumin } \\
\text { (mg/dL) }\end{array}$} & \multirow{2}{*}{$\begin{array}{l}\text { APACHE } \\
\text { II score }\end{array}$} & \multirow{2}{*}{$\begin{array}{l}\text { SOFA } \\
\text { score }\end{array}$} & \multirow[t]{2}{*}{ Survival } \\
\hline & & & Baseline & Peak & Baseline & $\overline{\text { Peak }}$ & Baseline & Peak & Baseline & Peak & & & & \\
\hline 5 & 76 & $M$ & 0.4 & 3.8 & 0.4 & 1.0 & 0.1 & 0.1 & 0.2 & 0.3 & 1.9 & 20 & 6 & Died on D57 \\
\hline 6 & 60 & $\mathrm{~F}$ & 9.6 & 12.9 & 1.7 & 1.7 & 0.3 & 0.4 & 0.4 & 0.5 & 3.5 & 16 & 4 & Alive \\
\hline 8 & 81 & $\mathrm{~F}$ & 18.9 & 51.2 & 0.7 & 1.3 & 0.4 & 0.9 & 0.3 & 0.4 & 3.2 & 16 & 4 & Alive \\
\hline 13 & 45 & M & 30.9 & 25.2 & 1.2 & 2.2 & 1.1 & 0.9 & 0.2 & 0.4 & 2.7 & 6 & 3 & Alive \\
\hline
\end{tabular}

Abbreviations: STC serum total cortisol, SaC salivary cortisol, CFC calculated free cortisol, CBG cortisol binding globulin, $D$ day of severe sepsis, $M$ male, $F$ female, APACHE II Acute Physiology and Chronic Health Evaluation II, SOFA Sequential Organ Failure Assessment, $D$ the day after the diagnosis of severe sepsis, GCC glucocorticoid 

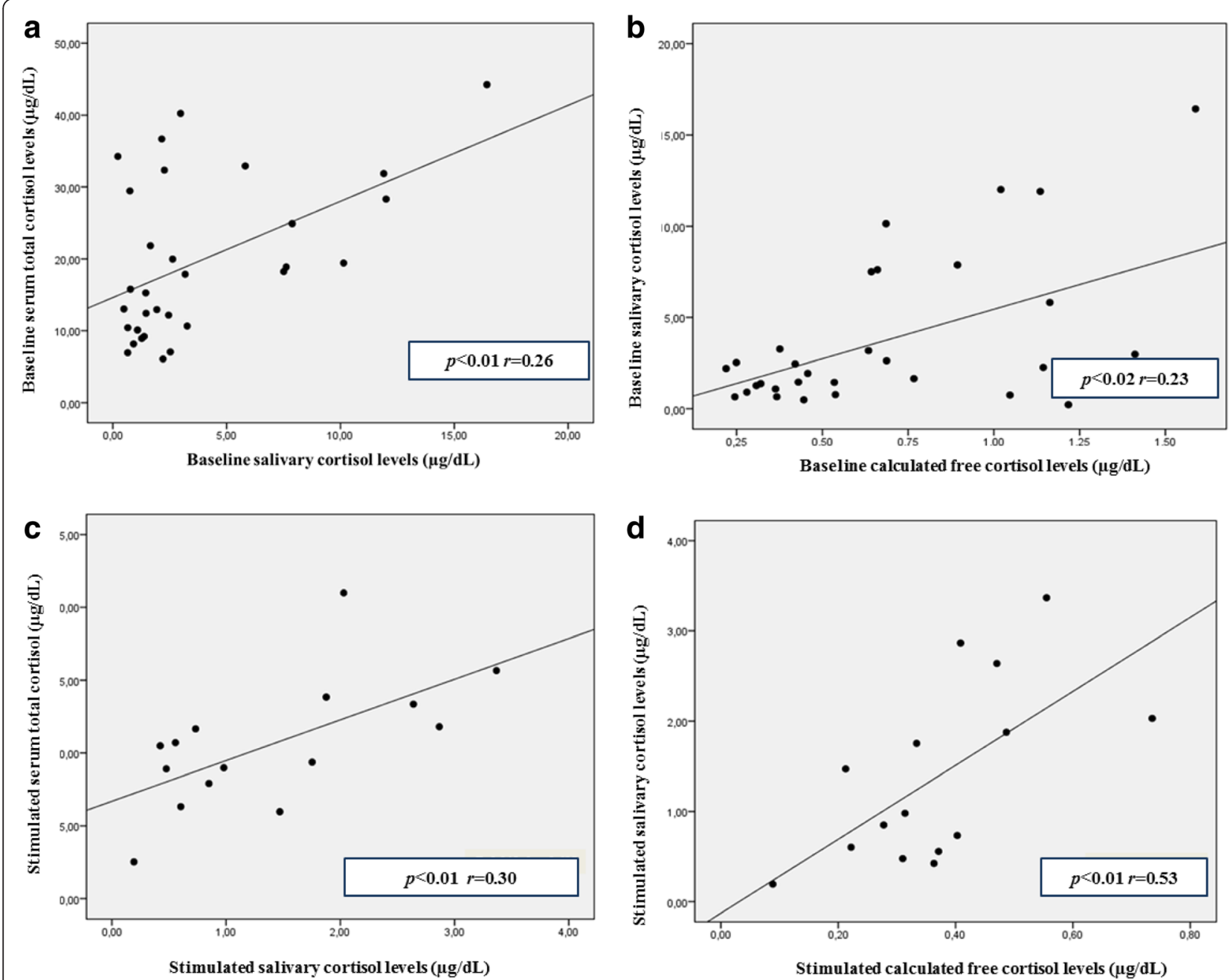

Fig. 2 Correlations between STC, SaC and CFC in baseline and stimulated conditions

$[12,30,31]$. And we have also shown that the CBG levels did not show an increase after ACTH stimulation.

As expected, stimulated STC levels were found to be lower in patients with SS who had low baseline STC levels than in healthy group. cFC formula strongly depends on STC, so similar tendency with STC and cFC levels is not surprising. One of the major limitations of the present study is that we have determined cFC levels by using STC and CBG measurements. So, cFC strongly reflects variations on STC and CBG levels. This explains the lower $\mathrm{CFC}$ levels obtained in SS subgroups than in the controls. If we could directly measure FC levels in patients with SS, we could do more certain judgement whether $\mathrm{SaC}$ levels better reflect or not serum FC levels.

Higher APACHE II and SOFA scores reflect worse clinical outcome. In many studies, SOFA or both SOFA and APACHE II scores were found to be related to mortality $[9,25,29]$. In the present study, the patients who did not survive also had higher APACHE II and SOFA scores. Although APACHE II and SOFA scores were not affected by the presence of low or high baseline STC levels, they were found to be related to mortality.

There was a significant correlation between $\mathrm{SaC}$ and $\mathrm{STC}$ and $\mathrm{SaC}$ and cFC. The relationship between $\mathrm{SaC}$ and STC is nonlinear; after a rapid increase in STC first, the serum CBG becomes saturated. CBG is saturated at a STC of 16 to $18 \mu \mathrm{g} / \mathrm{dL}$. After STC exceeds CBG saturation, the free portion of cortisol increases [32]. It could explain why $\mathrm{SaC}$ and $\mathrm{CFC}$ levels do not increase simultaneously.

\section{Conclusions}

In conclusion, SS is associated with increased $\mathrm{SaC}$, but decreased cFC levels when baseline STC is assumed to be sufficient. When STC level is assumed to be insufficient, $\mathrm{SaC}$ levels remain unchanged, but cFC levels are decreased. Lower STC levels is not associated with increased mortality in patients with SS. Although STC 
levels are correlated with $\mathrm{SaC}$ and $\mathrm{cFC}$ levels in patients with SS, these findings should be validated in an independent cohort before considering $\mathrm{SaC}$ or $\mathrm{cFC}$ instead of STC for diagnosing AI in patients with SS.

\begin{abstract}
Abbreviations
ACCP: American College of Chest Physicians; ACTH: adrenocorticotrophic hormone; Al: adrenal insufficiency; APACHE II: Acute Physiology and Chronic Health Evaluation II; CBG: cortisol binding globulin; CFC: calculated free cortisol; Cl: critical illness; CIRCl: critical illness related adrenal insufficiency; COPD: chronic obstructive pulmonary disease; D1: first day of diagnosis of severe sepsis; D28: 28th day of diagnosis of severe sepsis; D7: 7th day of diagnosis of severe sepsis; DM: diabetes mellitus; El: Enzyme immunassay; FC: free cortisol; HPA: Hypothalamus-pituitary-adrenal; ICU: Internal Medicine Intensive Care Unit; RAl: relative adrenal insufficiency; SaC: salivary cortisol; SCCM: Society of Critical Care Medicine; SOFA: Sequential Organ Failure Assessment; SPSS: Statistical Package for Social Sciences; SS: severe sepsis; STC: serum total cortisol.
\end{abstract}

\section{Competing interests}

The authors declare that they have no competing interests.

\section{Authors' contributions}

GE participated in the design and writing of the study and performed statistical analysis. ZK participated in the writing and proofreading of the study. FT participated in the design of the study and collected the data. KU is the keeper of the project, conceived the study and carried out the process of Erciyes University Council of Scientific Investigations. MS participated in the data collection and management of the patients and collected the written informed consents. MD participated in the data collection and consulted the patients in their courses of severe sepsis. FK conceived of the study and participated in its design and coordination. All authors read and approved the final manuscript.

\section{Acknowledgements}

The grant of this study was supported by the Erciyes University Council of Scientific Investigations (project code: TST-10-2929).

Written informed consent was obtained from the patients/or their first-degree relatives for the publication of their individual details and accompanying images in this manuscript. The consent form is held by the authors in the patients' clinical notes and is available for review by the Editor-in-Chief.

This manuscript was partly reported as a poster presentation at ICE/ENDO 2014 (June 21-24 in Chicago, IL,USA).

\section{Author details}

'Department of Endocrinology and Metabolism, Erciyes University Medical School, 38039 Kayseri, Turkey. ${ }^{2}$ Department of Internal Medicine, Erciyes University Medical School, Kayseri, Turkey. ${ }^{3}$ Department of Infectious Diseases, Erciyes University Medical School, Kayseri, Turkey.

\section{Received: 17 June 2015 Accepted: 30 December 2015}

\section{Published online: 08 January 2016}

\section{References}

1. Venkatesh B, Cohen J, Hickman I, Nisbet J, Thomas P, Ward G, et al. Evidence of altered cortisol metabolism in critically ill patients: a prospective study. Intensive Care Med. 2007;33(10):1746-53.

2. Venkataraman S, Munoz R, Candido C, Witchel SF. The hypothalamicpituitary-adrenal axis in critical illness. Rev Endocr Metab Disord. 2007:8(4):365-73.

3. Annane $D$, Bellissant E. Prognostic value of cortisol response in septic shock. JAMA. 2000:284(3):308-9.

4. Hamrahian $\mathrm{AH}$, Oseni TS, Arafah BM. Measurements of serum free cortisol in critically ill patients. N Engl J Med. 2004;350(16):1629-38.

5. Widmer IE, Puder JJ, König C, Pargger H, Zerkowski HR, Girard J, et al. Cortisol response in relation to the severity of stress and illness. J Clin Endocrinol Metab. 2005:90(8):4579-86.

6. Marik PE, Pastores SM, Annane D, Meduri GU, Sprung CL, Arlt W, et al. Recommendations for the diagnosis and management of corticosteroid insufficiency in critically ill adult patients: consensus statements from an international task force by the American College of Critical Care Medicine. Crit Care Med. 2008:36(6):1937-49.

7. Rothwell PM, Lawler PG. Prediction of outcome in intensive care patients using endocrine parameters. Crit Care Med. 1995:23(1):78-83.

8. Streeten $\mathrm{DH}$. What test for hypothalamic-pituitary-adrenocortical insufficiency? Lancet. 1999:354(9174):179-80.

9. Annane D, Maxime V, Ibrahim F, Alvarez JC, Abe E, Boudou P. Diagnosis of adrenal insufficiency in severe sepsis and septic shock. Am J Respir Crit Care Med. 2006;174(12):1319-26.

10. Dickstein G. High-dose and low-dose cosyntropin stimulation tests for diagnosis of adrenal insufficiency. Ann Intern Med. 2004;140(4):312-3.

11. Cooper MS, Stewart PM. Corticosteroid insufficiency in acutely ill patients. N Engl J Med. 2003;348(8):727-34.

12. Hammond GL, Smith CL, Underhill DA. Molecular studies of corticosteroid binding globulin structure, biosynthesis and function. J Steroid Biochem Mol Biol. 1991;40(4-6):755-62.

13. Coolens JL, Van Baelen H, Heyns W. Clinical use of unbound plasma cortisol as calculated from total cortisol and corticosteroid-binding globulin. J Steroid Biochem. 1987;26(2):197-202.

14. Ho JT, Al-Musalhi H, Chapman MJ, Quach T, Thomas PD, Bagley CJ, et al. Septic shock and sepsis: a comparison of total and free plasma cortisol levels. J Clin Endocrinol Metab. 2006;91(1):105-14.

15. Arafah BM, Nishiyama FJ, Tlaygeh $\mathrm{H}$, Hejal R. Measurement of salivary cortisol concentration in the assessment of adrenal function in critically ill subjects: a surrogate marker of the circulating free cortisol. J Clin Endocrinol Metab. 2007:92(8):2965-71.

16. Cohen J, Venkatesh B, Galligan J, Thomas P. Salivary cortisol concentration in the intensive care population: correlation with plasma cortisol values. Anaesth Intensive Care. 2004;32(6):843-5.

17. Gozansky WS, Lynn JS, Laudenslager ML, Kohrt WM. Salivary cortisol determined by enzyme immunoassay is preferable to serum total cortisol for assessment of dynamic hypothalamic-pituitary-adrenal axis activity. Clin Endocrinol (Oxf). 2005;63(3):336-41.

18. Schindhelm RK, van de Leur JJ, Rondeel JM. Salivary cortisol as an alternative for serum cortisol in the low-dose adrenocorticotropic hormone stimulation test? J Endocrinol Invest. 2010:33(2):92-5.

19. Deutschbein T, Unger N, Mann K, Petersenn S. Diagnosis of secondary adrenal insufficiency: unstimulated early morning cortisol in saliva and serum in comparison with the insulin tolerance test. Horm Metab Res. 2009:41(11):834-9.

20. Bone RC, Balk RA, Cerra FB, Dellinger RP, Fein AM, Knaus WA, et al. Definitions for sepsis and organ failure and guidelines for the use of innovative therapies in sepsis. The ACCP/SCCM Consensus Conference Committee. American College of Chest Physicians/Society of Critical Care Medicine Chest. 1992:136(5 Suppl):e28

21. Karaca Z, Lale A, Tanriverdi F, Kula M, Unluhizarci K, Kelestimur F. The comparison of low and standard dose ACTH and glucagon stimulation tests in the evaluation of hypothalamo-pituitary-adrenal axis in healthy adults. Pituitary. 2011:14(2):134-40.

22. Knaus WA, Draper EA, Wagner DP, Zimmerman JE. APACHE ॥: a severity of disease classification system. Crit Care Med. 1985;13(10):818-29.

23. Vincent JL, Moreno R, Takala J, Willatts S, De Mendonça A, Bruining $H$, et al. (Sepsis-related Organ Failure Assessment) score to describe organ dysfunction/failure. On behalf of the Working Group on Sepsis-Related Problems of the European Society of Intensive Care Medicine. Intensive Care Med. 1996;22(7):707-10

24. Topkas E, Keith P, Dimeski G, Cooper-White J, Punyadeera C. Evaluation of saliva collection devices for the analysis of proteins. Clin Chim Acta. 2012 413(13-14):1066-70.

25. Yildiz O, Tanriverdi F, Simsek S, Aygen B, Kelestimur F. The effects of moderate-dose steroid therapy in sepsis: a placebo-controlled, randomized study. J Res Med Sci. 2011;16(11):1410-21.

26. Aygen $B$, Inan M, Doganay M, Keleştimur F. Adrenal functions in patients with sepsis. Exp Clin Endocrinol Diabetes. 1997;105(3):182-6.

27. Venkatesh B, Mortimer $\mathrm{RH}$, Couchman B, Hall J. Evaluation of random plasma cortisol and the low dose corticotropin test as indicators of adrenal secretory capacity in critically ill patients: a prospective study. Anaesth Intensive Care. 2005;33(2):201-9.

28. Jenkins $\mathrm{RC}$, Ross RJM. The endocrinology of the critically ill: current opinion. Curr Opin Endocrinol Diab. 1996;3:138-45. 
29. Yildiz O, Doganay M, Aygen B, Güven M, Keleştimur F, Tutus A. Physiological-dose steroid therapy in sepsis. Crit Care. 2002;6(3):251-9.

30. Beishuizen A, Thijs LG, Vermes I. Patterns of corticosteroid binding globulin and the free cortisol index during septic shock and multitrauma. Intensive Care Med. 2001;27(10):1584-91.

31. Lin HY, Muller YA, Hammond GL. Molecular and structural basis of steroid hormone binding and release from corticosteroid-binding globulin. Mol Cell Endocrinol. 2010;316(1):3-12.

32. Aardal $\mathrm{E}, \mathrm{Holm}$ AC. Cortisol in saliva-reference ranges and relation to cortisol in serum. Eur J Clin Chem Clin Biochem. 1995;33(12):927-32.

Submit your next manuscript to BioMed Central and we will help you at every step:

- We accept pre-submission inquiries

- Our selector tool helps you to find the most relevant journal

- We provide round the clock customer support

- Convenient online submission

- Thorough peer review

- Inclusion in PubMed and all major indexing services

- Maximum visibility for your research

Submit your manuscript at www.biomedcentral.com/submit
Biomed Central 